\title{
Insulin amyloid fibrils form inclusion complex with molecular iodine: \\ a misfolded protein as a nanoscale scaffold
}

\author{
Wojciech Dzwolak $^{\text {a,b }}$ \\ ${ }^{a}$ Institute of High Pressure Physics, Polish Academy of Sciences, Sokolowska 29/37, 01-142 Warsaw, Poland \\ ${ }^{\mathrm{b}}$ Department of Chemistry, Warsaw University, Pasteura 1, 02-093 Warsaw, Poland \\ Phone: +48 22888 0237; Fax: +48 22632 4218; \\ E-mail:wdzwolak@unipress.waw.pl
}

Supporting Information

FT-IR spectra of the iodine-amyloid complex titrated with DMSO.



$\begin{array}{llllllll}1700 & 1680 & 1660 & 1640 & 1620 & 1600 & 1580\end{array}$

Wavenumber $\left[\mathrm{cm}^{-1}\right]$
Supplementary Figure 1. The infrared amide I band spectra of insulin amyloid titrated with DMSO (each spectrum is labeled with the corresponding DMSO weight percent). The spectra are the source data used for plotting the IR curve in Figure 5 of the manuscript. When the DMSO content reaches $90 \mathrm{wt}$ \% , a broad featureless band centered at $1661 \mathrm{~cm}^{-1}$ shows up at the expense of the spectral component at $1625 \mathrm{~cm}^{-1}$, which is a consequence of unfolding of $\beta$ strands into the DMSO-solvated random coil (Dzwolak et al. Biochemistry 45 (2006) 8143-8151). Heavy water was used in all amyloid preparations instead of light water because of the spectral overlap with the amide I band. In order to justify comparison of the titration-curves shown in Figure 5 of the manuscript, identical experimental conditions were maintained: e.g. in order to use the uniform protein concentration $(0.3 \mathrm{wt}$. \%) spectral pathlengths were varied between $0.1 \mathrm{~mm}$ for FT-IR and $2 \mathrm{~mm}$ for fluorescence and light scattering measurements. 